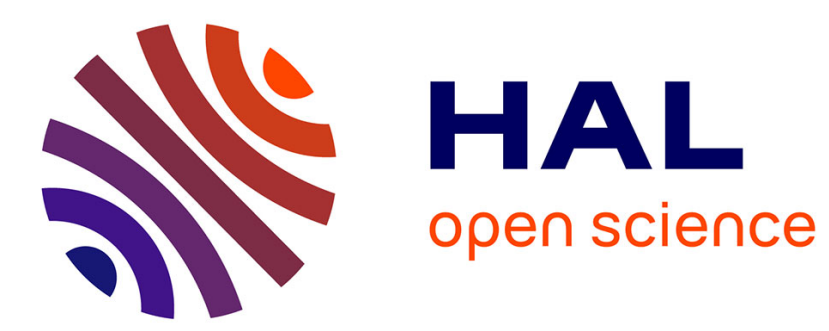

\title{
Le Bois et l'aménagement intérieur.
}

Pierre Parisot

\section{- To cite this version:}

Pierre Parisot. Le Bois et l'aménagement intérieur.. Revue forestière française, 2004, 56 (sp), pp.145148. 10.4267/2042/5133. hal-03449281

\section{HAL Id: hal-03449281 \\ https://hal.science/hal-03449281}

Submitted on 25 Nov 2021

HAL is a multi-disciplinary open access archive for the deposit and dissemination of scientific research documents, whether they are published or not. The documents may come from teaching and research institutions in France or abroad, or from public or private research centers.
L'archive ouverte pluridisciplinaire HAL, est destinée au dépôt et à la diffusion de documents scientifiques de niveau recherche, publiés ou non, émanant des établissements d'enseignement et de recherche français ou étrangers, des laboratoires publics ou privés. 


\section{LE BOIS DANS TOUTES SES FACETTES}

\section{LE BOIS ET L'AMÉNAGEMENT INTÉRIEUR}

\section{Pierre Parisot}

La décoration intérieure d'aujourd'hui, véritable reflet de la personnalité et du style de vie des populations qui composent la société, s'est fortement modifiée au fil des ans pour atteindre une dimension économique considérable, grâce à l'élévation générale du niveau culturel et aux outils mis à la disposition de tous ceux qui cherchent des idées, qu'il s'agisse de revues, d'expositions sur la maison et les objets, ou de magasins spécialisés sur le sujet. Les efforts pour réussir sa décoration sont considérables et le temps ainsi que les budgets qu'on y consacre sont élevés ; il faut dire que la décoration intérieure fait partie de la qualité de la vie et réussir sa décoration c'est avoir créé un certain type d'ambiance dans laquelle on se reconnaît et on se sent bien, c'est aussi pour tous les professionnels qui travaillent sur le sujet, que ce soit les architectes d'intérieurs, les designers ou les agenceurs, le talent de savoir concilier l'émotionnel et le fonctionnel.

\section{LE BOIS, MATÉRIAU d’EXCELLENCE POUR L’AMÉNAGEMENT INTÉRIEUR ET LA dÉCORATION}

Les propriétés intrinsèques du bois, à savoir tout d'abord son aspect qui joue un rôle primordial dans la décoration mais aussi sa facilité de mise en œuvre qui ne nécessite que des outils simples, sa bonne isolation thermique, son bon comportement acoustique, sont autant de points forts qui font du bois un matériau d'excellence pour la qualité des aménagements intérieurs, aussi bien du point de vue fonctionnel que sensoriel.

Le bois contribue à notre bien-être, parfois même sans que nous en soyons parfaitement conscients : tout d'abord par la variabilité de ses couleurs : on constate en effet que nous sommes fortement influencés par les couleurs qui évoluent au gré des modes et des tendances passant ainsi de tons plus sombres dans les périodes difficiles et plus austères, à des couleurs claires et des tons pastel dans les périodes plus gaies ou plus romantiques. Le toucher est lui aussi important et, dans ce domaine, le bois possède un avantage certain par rapport à d'autres matériaux ; on remarque que le toucher est essentiel dans le choix d'un matériau de décoration et tout le monde peut se rendre compte du geste naturel que nous faisons pour apprécier un tissu ou encore prendre possession d'un meuble. L'odeur de certains bois est aussi très appréciée et contribue à la qualité de vie des habitations.

La variabilité de ses décors est infinie grâce aux nombreuses essences qui existent et qui diffèrent entre elles selon les régions où les arbres ont poussé, ou encore par les modes de débit et d'usinage utilisés pour sa transformation, laissant ainsi un champ très vaste pour la créativité de nos architectes et designers.

Son origine biologique enfin, plutôt rassurante par les effets bénéfiques sur notre santé et sur les impacts positifs pour notre environnement. 


\section{LES GRANDES ÉVOLUTIONS DANS L’AMÉNAGEMENT INTÉRIEUR ET LA DÉCORATION}

Même si nous remontons très loin dans l'histoire, nous pouvons constater que le bois a toujours joué un rôle essentiel dans l'aménagement intérieur des hommes ; qu'il s'agisse des châteaux, des manoirs ou des grandes demeures, des maisons de paysans ou d'ouvriers, le bois est toujours présent. Certes, les essences et les styles diffèrent selon les époques, les régions et la richesse des populations, mais le bois constitue toujours l'essentiel de la décoration et de l'aménagement des intérieurs de notre habitat.

On assiste cependant à une rupture importante à une époque que l'on peut situer fin du XIX début du XXe siècle avec l'avènement de l'industrialisation qui a favorisé l'emploi du métal puis un peu plus tard les matériaux de synthèse avec le développement de la chimie.

Le mouvement "Art déco" des années 1930 innove en introduisant un raffinement formel et technique dans la décoration intérieure, mettant le bois en valeur avec des mobiliers élégants aux lignes courbes discrètement galbées, utilisant largement les bois cintrés, et les placages de bois précieux (Amarante, Bois de violette, Ébène de Macassar). Cette époque est marquée par de grands ébénistes comme Jacques Ruhlmann, et des industriels de génie comme Baumann et Thonet. Les bois les plus utilisés sont alors le Palissandre, le Noyer, le Frêne et le Poirier.

Plus près de nous, on constate un vrai changement des comportements, dans la période des années 1970-80 qui peuvent être considérées comme un tournant de l'histoire contemporaine avec le mouvement de mai 1968, qui voit la révolte de la génération de l'après-guerre et le premier choc pétrolier qui nous fait prendre conscience brutalement de notre fragilité en termes d'énergie.

Après les paillettes des "trente glorieuses", s'opère un grand retour à des valeurs sûres accompagné d'un certain repli sur soi-même, on invente même un mot nouveau, le "cocooning" qui traduit le désir de vivre dans un intérieur confortable où il fait bon s’isoler ; le confort chez soi va se trouver ainsi privilégié. C'est à cette époque que l'on constate alors un véritable engouement pour le bois "massif", dans les meubles et la décoration ; c'est aussi l'explosion du développement de la maison individuelle qui favorise l'emploi du bois et renforce cette notion de "cocooning".

Ce retour aux valeurs sûres que nous allons puiser dans les références au passé va se traduire par la référence aux styles traditionnels et rustiques revus et corrigés pour être mieux adaptés à nos modes de vie et aux nouvelles contraintes de l'industrialisation. Les bois les plus appréciés sont alors le Chêne avec les meubles de style (en particulier Louis XIII et Louis XIV), et les meubles rustiques, puis le Merisier avec le style Louis-Philippe qui va perdurer pendant plusieurs années.

C'est aussi l'époque où se développe un certain goût pour le rustique contemporain avec l'emploi de l'Orme qui va séduire toute une génération avec des créateurs comme Pierre Chapo et des marques commerciales comme "Regain" mais qui disparaîtra rapidement suite aux difficultés d'approvisionnement provoquées par la maladie des Ormes. Cette forte industrialisation va conduire aussi bon nombre d'entreprises à s'approvisionner en feuillus aux États-Unis ou au Canada pour les Chênes, Merisiers, Aulnes et Ormes, ou encore en Indonésie pour le Ramin qui permettra les plus belles imitations de Merisier.

\section{L'AMÉNAGEMENT INTÉRIEUR D'AUJOURD'HUI : TENDANCES LOURDES OU EFFETS DE MODE ?}

Le nouveau contexte économique, la modification de nos modes de vie, beaucoup plus tournés vers l'extérieur avec le développement du sport et des voyages, ont entraîné un goût plus prononcé pour des intérieurs plus gais et ont amené la mode des résineux, que ce soit en 
meuble ou en revêtements muraux ; autre formule économique mais aussi très en accord avec le style de vie actuelle, le meuble en kit, c'est-à-dire le meuble à emporter et à monter soimême, proposé par le géant suédois, Ikea. Ces meubles, les plus souvent réalisés à partir de résineux, vont accroître et démocratiser l'emploi du bois dans la maison, et être très appréciés des jeunes ménages.

Nous passons ainsi d'une époque où nos intérieurs étaient fortement chargés en moulures, en bois sculptés ou tournés, utilisant largement des bois aux finitions sombres renforcées par les "patines antiquaires", pour revenir à des décorations plus contemporaines qui vont peu à peu s'imposer dans un style plus dépouillé avec des lignes plus sobres que certains designers comme Philippe Starck vont largement démocratiser.

Globalement, on peut dire qu'aujourd'hui, la décoration intérieure fait appel à une plus grande variété d'essences : ainsi le Hêtre, le Frêne, le Bouleau, et d'une façon générale toutes les essences claires ont gagné des parts de marché importantes avec des finitions faisant largement appel à la couleur, que ce soit pour les pièces d'habitation principales ou dans les cuisines, ou même encore dans les salles de bain. Les designers et décorateurs aiment travailler sur les associations de bois de couleurs différentes et jouer sur les contrastes naturels des bois. Ils vont aussi réaliser des mélanges de matériaux, en particulier le bois avec le verre qui va donner sa touche de clarté et de transparence, ou encore le fer forgé apportant aux produits bois ce côté un peu "rétro" empreint de légèreté et évocateur d'un certain romantisme.

La décoration d'aujourd'hui tire aussi largement son inspiration du voyage ; l'exotisme tient une place importante de même qu'on y retrouve ce côté "Zen" que recherchent les jeunes générations et que l'on retrouve dans beaucoup de revues spécialisées.

Ce qui est apprécié, c'est la simplicité mais aussi et surtout l'authenticité : fini les imitations de toutes sortes; on préfère un décor plus simple mais surtout plus vrai. Les revêtements muraux ont eux aussi fortement évolué avec l'avènement des lambris dans des finitions aux tons pastel, grâce en particulier aux lasures qui ont l'avantage d'apporter de la couleur tout en laissant le bois apparent. Les finitions connaissent une profonde évolution : elles sont à la fois plus légères et plus travaillées ; ainsi l'aspect ciré est plus recherché que le brillant des vernis traditionnels. Elles mettent en jeu des teintes et des couleurs et réclament souvent des traitements particuliers au niveau des états de surface conduisant à des effets vieillis très recherchés : on trouve ainsi des chênes brossés ou brûlés avant d'être recouverts de teintes, ou encore ces finitions de type peintures vieillies très appréciées et qui rappellent que le meuble a son histoire, qu'il a vécu et qu'il provient peut-être d'une de ces nombreuses brocantes qui fleurissent aujourd'hui un peu partout...

On considère souvent que la tendance actuelle est aux mélanges : il n’y a pas une décoration type qui dicte sa loi ; chacun invente et imagine sa propre décoration ; on trouvera aussi bien des produits faisant référence au passé dans un intérieur très contemporain, en particulier dans le salon, la cuisine ou la salle de bain, trois pièces moins imprégnées de tradition et qui permettent une plus grande liberté dans la décoration.

Quand on aborde la décoration intérieure, il n'est pas possible de ne pas parler du "bricolage", véritable phénomène de notre société moderne où le bois occupe une place de choix ; d'après les sources du CETELEM, la décoration intérieure représenterait plus de $20 \%$ de l'ensemble du chiffre d'affaires du marché du bricolage, avec des progressions annuelles de $6 \%$ à $7 \%$. En dehors des aspects économiques, le principe même du bricolage est intéressant dans la décoration intérieure, par la liberté qu’il permet dans les projets imaginés par chacun et le plaisir qu'il procure pour ceux qui osent les réaliser. 


\section{QUEL SERA LE FUTUR POUR LA DÉCORATION INTÉRIEURE ? SERA-T-IL TRÈS DIFFÉRENT DU PRÉSENT ?}

Si nous nous référons aux pronostics annoncés par les innombrables bureaux de tendances qui se sont développés ces dernières années et qui se sont souvent avérés erronés, on peut en conclure qu'il est présomptueux voire impossible de savoir quels seront les goûts des futures générations compte tenu des nombreux facteurs socioculturels qui influencent ces goûts. Cependant, on peut se risquer à penser que le bois aura toujours une place de choix dans l'aménagement intérieur. En effet, si nous extrapolons les raisons qui aujourd'hui font apprécier le bois, on constate qu'il s'agit de tendances de fond comme le goût de l'authentique, et la recherche sécuritaire vis-à-vis de la santé qui fait préférer les matériaux naturels, ainsi qu'une forte prise de conscience pour l'écologie. Il semble bien que tous ces facteurs iront en s'amplifiant et ne sont pas près de disparaître.

Cette poussée écologique se traduit déjà par une recherche de bois et de finitions qui évoquent "le naturel" ; ainsi, après le succès des grandes essences traditionnelles de l'ébénisterie comme les Merisiers, les Noyers, et les Chênes, on va trouver des essences plus communes qui sont surtout appréciées par les lieux qu'elles évoquent et qui correspondent le plus souvent à leurs origines : ainsi le Sapin ou l'Épicéa évoque pour beaucoup les grandes forêts des Vosges ou du Nord de l'Europe ; le Pin, la pinède du bord de mer ; le Chêne, les forêts profondes et mystérieuses des contes de notre enfance...

On voit donc qu'une tendance actuelle en matière de bois est plus la préoccupation intuitive des utilisateurs sur l'origine et le lieu de croissance qu'ils évoquent dans leur imaginaire, que par les qualités intrinsèques que ce lieu confère au bois ; on comprend ainsi pourquoi le marché est de plus en plus sensible à la notion d'origine et pourquoi pas demain aussi aux notions d'écocertification et de traçabilité, à condition cependant que le langage technique adopte des modes de communication facilement compréhensibles pour le consommateur et explicables par le vendeur.

Vis-à-vis de ces évolutions probables, nous voyons que le bois a de réels points forts. Pourtant on peut estimer que les deux plus grands dangers seraient que le bois devienne un matériau banal sans aucune valeur affective, ou au contraire un matériau de luxe difficilement abordable pour la plupart des personnes qui l'apprécient ; c'est donc à tous les acteurs de la filière-bois qu'il appartient de trouver les solutions techniques mais aussi marketing pour que l'emploi du bois poursuive son développement et continue à agrémenter notre cadre de vie.

Directeur du Pôle Ameublement CENTRE TECHNIQUE DU BOIS

ET DE L'AMEUBLEMENT (CTBA)

10, avenue de Saint-Mandé F-75012 PARIS

(pierre.parisot@ctba.fr)

\section{LE BOIS ET L'RmÉNAgEmENT INTÉRIEUR [Résumé]}

La décoration intérieure d'aujourd'hui, véritable reflet de la personnalité et du style de vie des populations qui composent la société, s'est fortement modifiée au fil des ans pour atteindre une dimension économique considérable. Le bois est un matériau d'excellence pour la décoration intérieure. Après avoir évoqué les grandes évolutions du passé, les tendances lourdes ou les effets de mode de l'aménagement intérieur d'aujourd'hui sont abordés avant de s’interroger sur ce que pourra être la décoration dans le futur.

\section{UOOD AND INTERIOR DECORATION [Rbstract]}

Interior decorating, a true reflection of the personality and lifestyle of the populations that make up society, has changed considerably over time and is now a significant business activity. Wood is a superior material for interior decoration. The main developments of the past are first described, followed by both lasting trends and passing fads in contemporary interior decoration. Potential future developments in decoration are then considered. 